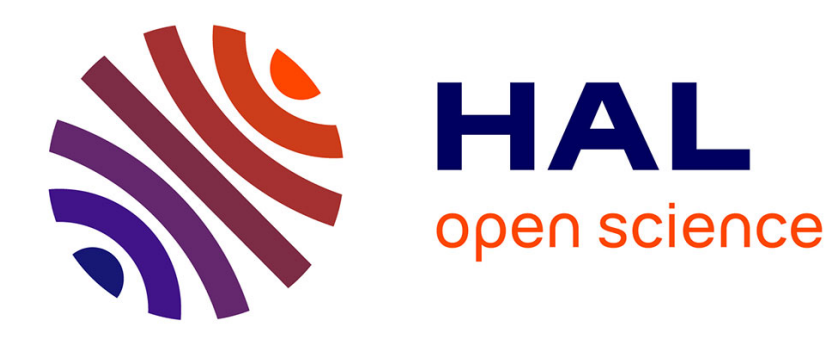

\title{
Influence of the dc laser heating on the optical beam deflection
}

\author{
S. Surnev, D. Ivanov
}

\section{To cite this version:}

S. Surnev, D. Ivanov. Influence of the dc laser heating on the optical beam deflection. Revue de Physique Appliquée, 1990, 25 (5), pp.457-462. 10.1051/rphysap:01990002505045700 . jpa-00246205

\section{HAL Id: jpa-00246205 https://hal.science/jpa-00246205}

Submitted on 1 Jan 1990

HAL is a multi-disciplinary open access archive for the deposit and dissemination of scientific research documents, whether they are published or not. The documents may come from teaching and research institutions in France or abroad, or from public or private research centers.
L'archive ouverte pluridisciplinaire HAL, est destinée au dépôt et à la diffusion de documents scientifiques de niveau recherche, publiés ou non, émanant des établissements d'enseignement et de recherche français ou étrangers, des laboratoires publics ou privés. 
Classification

Physics Abstracts

$78.20-44.50$

\title{
Influence of the dc laser heating on the optical beam deflection
}

\author{
S. Surnev and D. Ivanov \\ Department of Solid State Physics, Faculty of Physics, Sofia University, 5 A. Ivanov Blvd., Sofia 1126, \\ Bulgaria
}

(Reçu le 12 octobre 1989, révisé le 11 janvier 1990, accepté le 19 janvier 1990)

\begin{abstract}
Résumé. - Une étude de l'influence de l'échauffement permanent, obtenu par le rayonnement continu du laser, sur l'effet mirage est proposée. On a établi que l'échauffement continu provoque une diminution de l'amplitude du signal de l'effet mirage et une augmentation de sa phase. Ceci est dû à la variation de l'indice de réfraction et à la diffusivité thermique du milieu de réfraction (l'air) avec la température. Une expression théorique de la distribution de la température du milieu de réfraction est établie. Elle permet d'estimer l'influence de la température d'équilibre sur le signal de l'effet mirage. Des expériences avec des échantillons de quartz fondu et de sulfide de cadmium ont été effectuées montrant une forte dépendance de la température du milieu de réfraction de la conductivité thermique. Ceci est en bon accord avec les résultats théoriques. Les calculs montrent aussi qu'une variation de $3 \mathrm{~K}$ provoque un changement de l'amplitude du signal d'environ $1 \%$ et une variation de sa phase d'environ $1^{\circ}$.
\end{abstract}

\begin{abstract}
The influence of the dc laser heating on the optical beam deflection (OBD) has been studied. It was found that the dc heating causes a decrease in the OBD amplitude and an increase in the OBD phase. This is due to variations of the refractive index and thermal diffusivity of the deflecting medium (air) with temperature. A theoretical expression for the temperature distribution in the deflecting medium has been derived. It allows one to estimate the influence of the steady state temperature on the OBD signal. Experiments made with fused quartz and cadmium sulfide samples have shown a strong dependence of the temperature of the deflecting medium on the sample thermal conductivity that is in good agreement with theoretical results. Calculations also show that a variation of $3 \mathrm{~K}$ leads to an amplitude change of about $1 \%$ and a phase change of about $1^{\circ}$.
\end{abstract}

\section{Introduction.}

A number of new experimental methods for determining some thermo-optical material constants, based on the photothermal and photoacoustic effects, have been developed during the last decade. Among them very popular is the method of photothermal optical beam deflection (OBD) of a probe laser beam, known also as «mirage effect » [1-3]. In the OBD experiments the sample is periodically heated by a modulated (pump) laser beam. Thermally induced index-of-refraction of air causes a periodical deflection of a second (probe) laser beam moving parallel to and above the sample surface. The magnitude and the direction of this deflection is determined by the thermo-optical properties of the sample and the deflecting fluid (air).

In the modulated photothermal methods (including OBD) the time independent (dc) component of the laser heating is usually neglected, since the magnitude of the signal is described by the time dependent (ac) one. In a number of cases (poor heat conductors, high power laser beams), however, the dc heating may cause changes of the photothermal signal because of the variation of some temperature dependent optical and thermal parameters of the sample and the fluid. According to Opsal et al. [4] the local dc and ac heating in thermal wave experiments can produce temperature excursions in a broad interval, depending on the thermal characteristics of the sample. In their model temperature effects on the refractive index of the fluid and on the thermal conductivity of the sample are considered as a possible source of nonlinearities.

In the mirage effect method the parameters, which define the OBD signal, are the temperature derivative of the refractive index of the deflecting fluid and the thermal diffusivities of the fluid and the sample. Since these parameters are temperature dependent, such effects should be expected here 
too. When the thermal diffusivity of the sample varies slowly with temperature and the fluid is air the OBD signal temperature dependence is familiar [5] and studying the influence of the dc heating of the sample on the mirage effect characteristics is possible. Sources of the heating might be the pump laser beam or an additional $\mathrm{cw}$ laser beam. Taking into account these effects, caused by the heating, is important for the OBD method itself, as well as for the development of new photothermal methods.

\section{Experimental.}

The experimental setup is shown in figure 1. It consists of a transverse mirage effect scheme in air and a heating cw Ar laser. The sample is periodically heated by a He-Ne (pump) laser beam $(\lambda=632.8 \mathrm{~nm})$, modulated by a mechanical chopper at frequency $200 \mathrm{~Hz}$. The pump beam of power $30 \mathrm{~mW}$ is unfocussed (spot size $\sim 3 \mathrm{~mm}$ ) and directed at normal incidence to the sample surface. The periodical deflection of the probe He-Ne laser beam $(\lambda=632.8 \mathrm{~nm})$ of power $0.5 \mathrm{~mW}$ is detected by a silicon position sensor (SIEMENS-type BPX 48). The signal from the detector is fed to a PARC 5316 lock-in amplifier. Only the normal (to the sample surface) component of the deflection is measured, due to the large pump beam diameter.

The studied samples are a $5 \times 10 \times 10 \mathrm{~mm} \mathrm{CdS}$ crystal and a fused quartz specimen, mounted on a three dimensional moving stage. For better light absorption one of the sample surfaces is blackened and this is positioned at constant distance $z$ from the probe beam (probe beam offset). The thermal conductivities of the $\mathrm{CdS}$ and the fused quartz samples are $27.0 \mathrm{~W} \cdot \mathrm{m}^{-1} \cdot \mathrm{K}^{-1}$ and $1.44 \mathrm{~W} . \mathrm{m}^{-1} . \mathrm{K}^{-1}$, respectively [6].

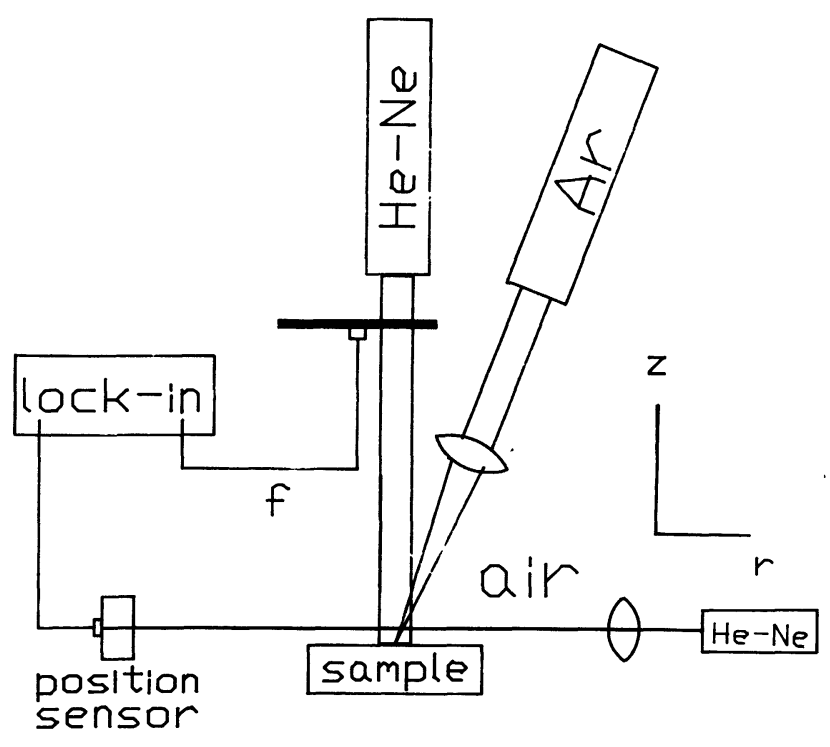

Fig. 1. - Schematic diagram of the experimental setup.
The dc heating of the sample is accomplished by a cw Ar laser beam $(\lambda=514.5 \mathrm{~nm})$ of power varying from $50 \mathrm{~mW}$ to $250 \mathrm{~mW}$, monitored with a powermeter. The beam, which falls nearly perpendicularly to the sample surface, is focused to a spot of approximately $400 \mu \mathrm{m}$ in diameter.

The probe beam with a diameter $\sim 100 \mu \mathrm{m}$ is adjusted very close to the heating beam spot center.

\section{Experimental results.}

Figure 2 presents a plot of the OBD amplitude and phase as a function of the probe beam offset $z$, measured for a fused quartz. Because of the finite size of the probe beam this offset is shifted from the surface by about $50 \mu \mathrm{m}$. The experimental curves, denoted 1 refer to the amplitude (Fig. 2a) and phase (Fig. 2b) of the signal before switching the dc heating on and those indicated with 2-to their values when the steady-state regime for the dc heating has been established. The power of the Ar laser beam, used for heating the sample, was set to $50 \mathrm{~mW}$. As seen the curves 1 demonstrate the typical dependence of the OBD signal on the probe beam offset : exponential for the amplitude and linear for the phase. The continuous lines in figure 2 represent these functions, fitted to the experimental data 1 by the least squares method. At small beam offsets the difference between the OBD amplitudes 1 and 2 is maximal. With increasing $z$ this difference decreases gradually. The behaviour of the OBD phase during the heating is similar to that before heating.

For the sake of convenience we introduce the terms relative amplitude and relative phase of the OBD signal, defined as the ratio (difference) of the steady-state values of the amplitude (phase) during and before the dc heating of the sample, respectively. Figure 3 represents the relative amplitude (Fig. 3a) and the relative phase (Fig. $3 b$ ), measured as a function of the heating beam power at beam offset $z=200 \mu \mathrm{m}$, for CdS and fused quartz. It is clear that the relative amplitude decreases with increasing the heating beam power. The rate of this decrease is much higher for the fused quartz sample than for the $\mathrm{CdS}$. Figure $3 \mathrm{~b}$ shows that the relative phase anticorrelates with the relative amplitude, in that it increases with increasing the laser power.

\section{Discussion.}

In order to explain the observed changes of the OBD signal, which occur during the dc laser beam heating of the sample, we need to know :

1) the temperature field in the deflecting fluid above the sample, created by the heating source, and ;

2) the influence of this field on the mirage effect. 

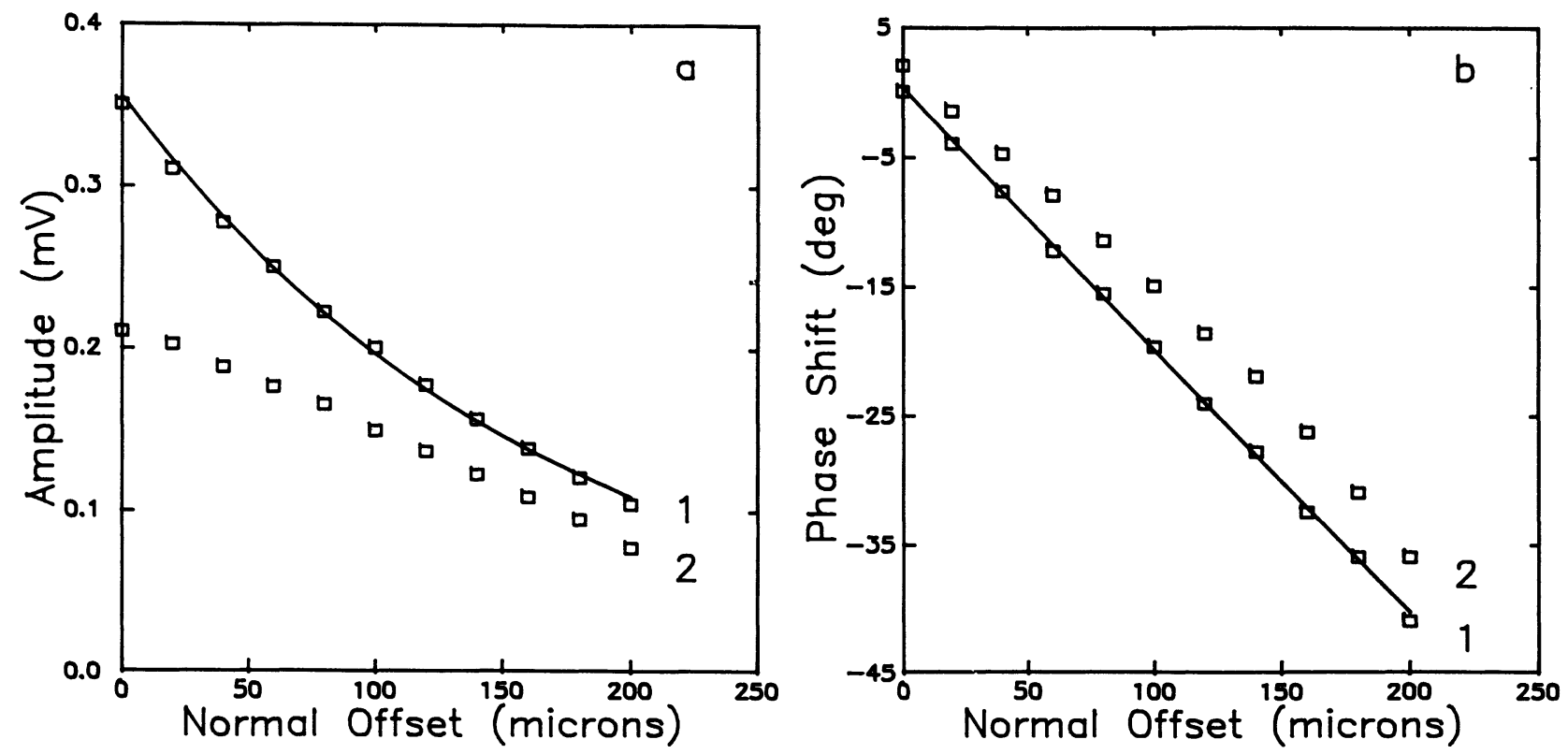

Fig. 2. - OBD amplitude (a) and phase (b), measured as a function of the probe beam offset for a fused quart/. I he curves denoted with 1 are related to the OBD signal before the heating and those denoted with 2-to the OBD signal during the heating (Ar laser power $P_{0}=50 \mathrm{~mW}$ ). Curves 1 are fitted by the least squares method.
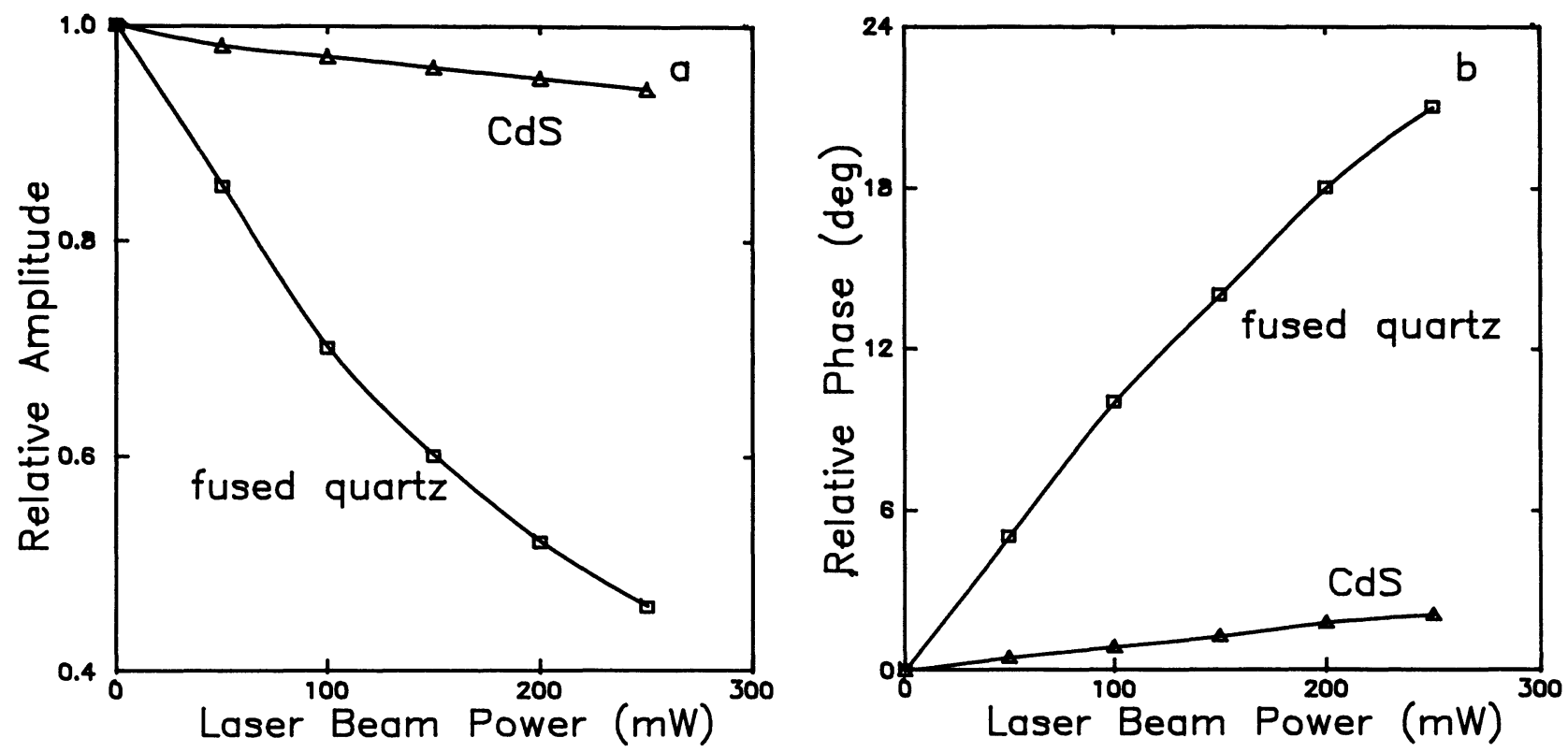

Fig. 3. - Relative OBD amplitude (a) and phase (b), measured as a function of the heating beam power for the samples of fused quartz $(\square)$ and CdS $(\Delta)$. Probe beam offset $z=200 \mu \mathrm{m}$.

4.1 Calculation OF THE STEADY-STATE TEMPERATURE DISTRIBUTION IN THE FLUID. - Under the experimental conditions, discussed in section 2 , we neglect the dc heating of the pump He-Ne laser beam (power density $\sim 4 \times 10^{3} \mathrm{~W} \cdot \mathrm{m}^{-2}$ ) compared to the $\mathrm{Ar}$ one (power density $\left.\sim 4 \times 10^{5}-2 \times 10^{6} \mathrm{~W} \cdot \mathrm{m}^{-2}\right)$. We consider the heating source situated at the sample surface $z=0$ (surface absorption) and its intensity distribution is assumed to be Gaussian :

$$
F(r)=F_{0} \exp \left(-\frac{r^{2}}{a^{2}}\right)
$$

where $F_{0}=\frac{P_{0}}{\pi a^{2}}$ is the intensity at the heating beam center $r=0, a$ is the Gaussian radius of the heating 
beam spot (defined as the distance from the spot center at which $F(r)$ decreases $e$-times), and $P_{0}$ is the power of the heating beam, which is assumed to be absorbed at the sample surface. The sample is considered as a thermally homogeneous, semi-infinite medium.

The increase in the temperature above its ambient value $T_{0}$ can be calculated from the steady-state thermal conductivity equation :

$$
\Delta T_{i}(r, z)=0, \quad i=s, f
$$

subject to the following boundary conditions :

$$
\begin{aligned}
& \left.T_{\mathrm{s}}(r, z)\right|_{z=0}=\left.T_{\mathrm{f}}(r, z)\right|_{z=0} \quad T_{\mathrm{s}, \mathrm{f}}(r, z) \underset{r \rightarrow \infty}{\longrightarrow} 0- \\
& -\left.k_{\mathrm{s}} \frac{\partial T_{\mathrm{s}}(r, z)}{\partial z}\right|_{==0}=-\left.h \cdot T_{\mathrm{s}}(r, z)\right|_{z=0}+F(r),
\end{aligned}
$$

where $\Delta$ denotes the Laplace operator, $k$ is the thermal conductivity, $h$ is the heat transfer coefficient and the subscripts $\mathbf{s}$ and $\mathrm{f}$ refer to the sample $(z<0)$ and the fluid $(z>0)$, respectively. The temperature distribution in the fluid can be obtained under the assumption that the loss of heat at any position above the illuminated area is proportional to the temperature at that position [7]. Because of the radial symmetry of the source we are looking for solutions of (2) in the form [8] :

$$
\begin{aligned}
& T_{\mathrm{s}}(r, z)=\int_{0}^{\infty} A(\lambda) \cdot \exp (\lambda \cdot z) \cdot J_{0}(\lambda \cdot r) \cdot \mathrm{d} \lambda \\
& T_{\mathrm{f}}(r, z)=\int_{0}^{\infty} B(\lambda) \cdot \exp (-\lambda \cdot z) \cdot J_{0}(\lambda \cdot r) \cdot \mathrm{d} \lambda
\end{aligned}
$$

where the functions $A(\lambda)$ and $B(\lambda)$ can be derived from the boundary conditions (3) and $J_{0}$ is the Bessel function of zero order. Substituting (4) and (5) in (3) we obtain :

$$
\begin{aligned}
& T_{\mathrm{f}}(r, z)=\frac{P_{0}}{2 \pi k_{\mathrm{s}}} \times \\
& \times \int_{0}^{\infty} \frac{\lambda \cdot \exp \left[-\left(\lambda^{2} a^{2} / 4+\lambda \cdot z\right)\right] \cdot J_{0}(\lambda \cdot r)}{\lambda+h / k_{\mathrm{s}}} \mathrm{d} \lambda .
\end{aligned}
$$

Figure 4 presents a plot of $T_{\mathrm{f}}(r, z)$ as a function of $z$, expressed in units of $a$, at two values of the heat transfer coefficient : $h=0 \mathrm{~W} \cdot \mathrm{m}^{-2} \cdot \mathrm{K}^{-1}$ (solid line) and $h=100 \mathrm{~W} \cdot \mathrm{m}^{-2} \cdot \mathrm{K}^{-1}$ (dashed line) at $r=0$, $P_{0}=100 \mathrm{~mW}, \quad k_{\mathrm{s}}=1 \mathrm{~W} \cdot \mathrm{m}^{-1} \cdot \mathrm{K}^{-1} \quad$ and $a=200 \mu \mathrm{m}$. Both curves differ by less than $2 \%$, which indicates that $h=0$ is a good approximation. Let us now consider the temperature distribution in the fluid, above the heating spot center $r=0$. In this

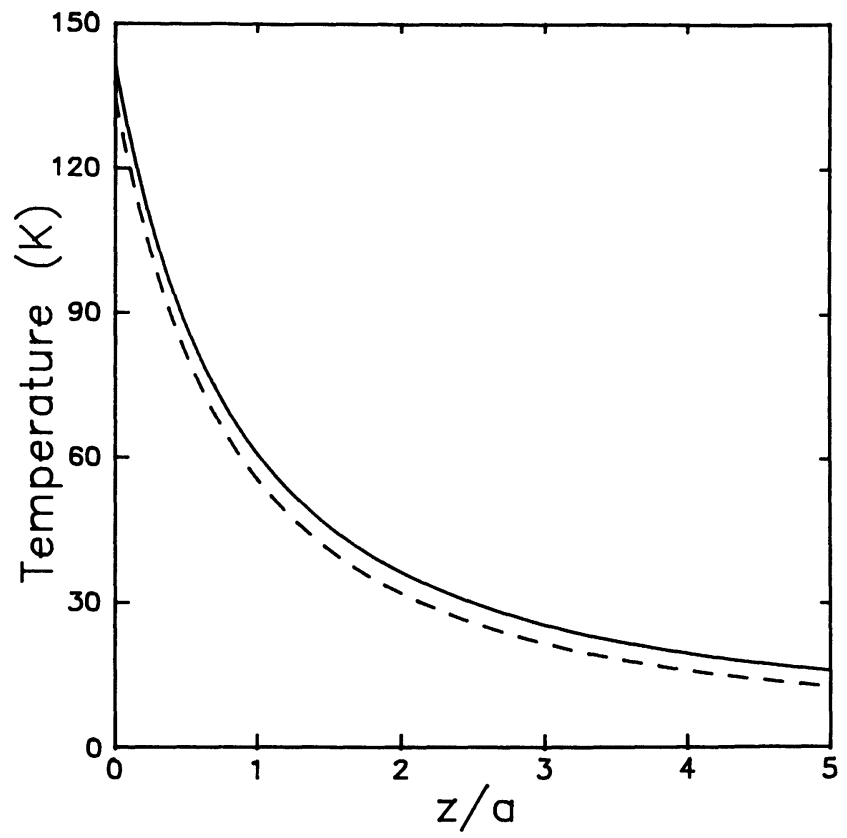

Fig. 4. - Plot of the steady state temperature $T_{\mathrm{f}}$ of the fluid (above the ambient temperature) calculated as a function of the probe beam offset $z$, expressed in units of the heating beam radius $a$. The solid curve refers to $h=0 \mathrm{~W} \cdot \mathrm{m}^{-2} \mathrm{~K}^{-1}$ and the dashed one- to $h=100 \mathrm{~W} \cdot \mathrm{m}^{-2} \mathrm{~K}^{-1}$. The values of the parameters are as follows : $a=200 \mu \mathrm{m}, P_{0}=100 \mathrm{~mW}, k=1 \mathrm{~W} \cdot \mathrm{m}^{-1} \cdot \mathrm{K}^{-1}$.

case the integration in (6) can be done and we finally obtain :

$$
T_{\mathrm{f}}(0, z)=\frac{P_{0}}{2 \sqrt{\pi} a k_{\mathrm{s}}} \cdot \exp \left(\frac{z^{2}}{a^{2}}\right) \cdot \operatorname{erfc}\left(\frac{z}{a}\right)
$$

where erfc is the complementary error function. Hence at a given fixed distance $z$ above the sample surface and a heating beam radius $a$ the steady-state temperature in the fluid is proportional to the heating laser power and inversely proportional to the thermal conductivity coefficient of the sample.

4.2 INFLUENCE OF THE dc HEATING ON OBD. - In view of the large pump beam spot size compared to that of the probe beam we can use the one dimensional model of mirage effect. For the case of thermally thick sample $\left(\ell_{\mathrm{s}} / \mu_{\mathrm{s}} \gg 1, \ell_{\mathrm{s}}\right.$ is the sample thickness and $\mu_{s}$ is the thermal diffusion length of the sample) and of photothermal saturation $\left(\beta_{s} \cdot \mu_{s} \gg 1, \beta_{s}\right.$ is the optical absorption coefficient of the sample) the amplitude $S$ and the phase $\phi$ of the OBD signal are given by [9] :

$$
\begin{aligned}
S & =S_{1}(\partial n / \partial T)_{\mathrm{f}}\left(\alpha_{\mathrm{s}} / \alpha_{\mathrm{f}}\right)^{1 / 2} \exp \left(-z / \mu_{\mathrm{f}}\right) \\
\phi & =\phi_{1}-z / \mu_{\mathrm{f}},
\end{aligned}
$$

where $S_{1}$ and $\phi_{1}$ are constants, $(\partial n / \partial T)_{\mathrm{f}}$ is the temperature derivative of the fluid's refractive index, $\alpha$ is the thermal diffusivity $\left[m^{2} . s^{-1}\right]$ 
$\left(\alpha=k /(\rho \cdot C)\right.$, where $\rho$ is the density $\left[\mathrm{kg} \cdot \mathrm{m}^{-3}\right]$ and $C$ is the specific heat $\left.\left[\mathrm{J} \cdot \mathrm{kg}^{-1} \mathrm{~K}^{-1}\right]\right) z$ is the probe beam offset, $\mu_{\mathrm{f}}=\sqrt{\alpha_{\mathrm{f}} /(\pi f)}$ is the thermal diffusion length of the fluid at modulation frequency $f$.

According to [5] the OBD signal in air, at constant air pressure, depends on the temperature $T\left(T=T_{0}+T_{\mathrm{f}}\right.$, where $T_{0}$ is the ambient temperature $)$ as follows :

$$
\begin{aligned}
& S=A \cdot T^{-2.75} \cdot \exp \left(-B \cdot T^{-0.75} \cdot z\right) \\
& \phi=C-B \cdot T^{-0.75} \cdot z,
\end{aligned}
$$

where $A, B$ and $C$ are constants. It is suggested [5] that $\mathrm{OBD}$ is temperature dependent through the refractive index and the thermal diffusivity of the fluid, provided that the properties of the sample are constant. If the fluid is treated as an ideal gas the refractive index varies as $T^{-2}$ and the thermal diffusivity as $T^{1.5}$ (or as $T^{1.46}$ in the case of air, as was experimentally confirmed in Ref. [7]).

If we substitute the fluid's temperature distribution (7), calculated in subsection 4.1, in (10) and (11) we obtain the dependence of OBD on the probe beam offset. Figure 5 shows a plot of the calculated OBD amplitude (Fig. 5a) and phase (Fig. 5b) as a function of the probe beam offset $z$ with (dashed line) and without (solid line) heating for $P_{0}=50 \mathrm{~mW}$, $k_{\mathrm{s}}=1 \mathrm{~W} \cdot \mathrm{m}^{-1} . \mathrm{K}^{-1}, \quad a=200 \mu \mathrm{m}, \quad T_{0}=300 \mathrm{~K}$, $A=2 \times 10^{6}, B=3 \times 10^{5}$ and $C=0$. Obviously, these curves are in good qualitative agreement with the experimental ones, presented in figure 2.

Let the amplitude and the phase of the OBD signal before heating $\left(T=T_{0}\right)$ are $S_{0}$ are $\phi_{0}$, respect- ively. By the time the steady-state regime during heating is established the temperature of the fluid has already increased to $T=T_{0}+T_{\mathrm{f}}$ and the amplitude and the phase have become $S$ and $\phi$, respectively. The relative amplitude and the relative phase of OBD then are :

$$
\begin{aligned}
S / S_{0}= & \left(\frac{T}{T_{0}}\right)^{-2.75} \exp \left[-z B\left(T^{-0.75}-T_{0}^{-0.75}\right)\right] \\
& \phi-\phi_{0}=-z B\left(T^{-0.75}-T_{0}^{-0.75}\right) .
\end{aligned}
$$

Figure 6 shows the calculated relative amplitude and phase as a function of the heating beam power for $k_{\mathrm{s}}=1 \mathrm{~W} \cdot \mathrm{m}^{-1} \cdot \mathrm{K}^{-1} \quad$ (curve 1) and $k_{\mathrm{s}}=20 \mathrm{~W} \cdot \mathrm{m}^{-1} \cdot \mathrm{K}^{-1}$ (curve 2) at $z=200 \mu \mathrm{m}$, $a=200 \mu \mathrm{m}, T_{0}=300 \mathrm{~K}$ and $B=5 \times 10^{5}$. As can be seen in figure 6 the increase in the heating power causes a decrease in the mirage amplitude (Fig. 6a) and an increase in the mirage phase (Fig. 6b). It is also evident that at lower sample thermal conductivity the degree of the amplitude decrease (phase increase) is higher. This explains the behaviour of the experimental OBD signal when increasing the heating beam power (Fig. 3).

A useful criterion showing the limits of application of the OBD method in the presence of dc laser beam heating can be derived. If we require the relative OBD amplitude and phase at a given beam offset to be more than $99 \%$ and less that $1^{\circ}$, respectively, then combining (12) and (13) we obtain :

$$
T_{\mathrm{f}}<3 \mathrm{~K}
$$

where $T_{\mathrm{f}}$ is the temperature rise of the fluid at this offset.
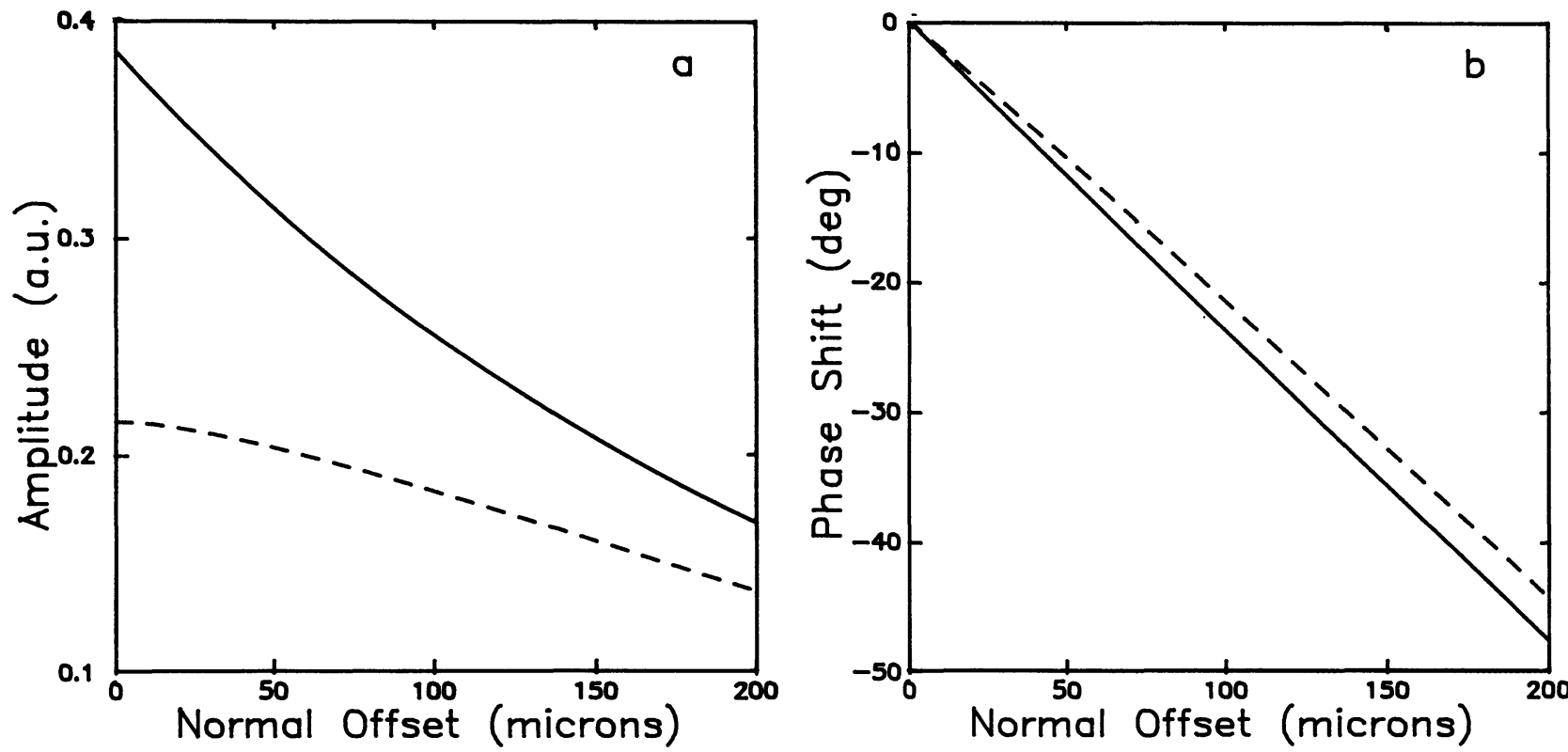

Fig. 5. - OBD amplitude (a) and phase (b) calculated as a function of probe beam offset $z$ during the heating (dashed line) and before the heating (solid line) at the following values of the parameters : $a=200 \mu \mathrm{m}, P_{0}=50 \mathrm{~mW}$, $k=1 \mathrm{~W} \cdot \mathrm{m}^{-1} \cdot \mathrm{K}^{-1}, A=2 \times 10^{6}, B=3 \times 10^{5}, C=0$ and $T_{0}=300 \mathrm{~K}$. 

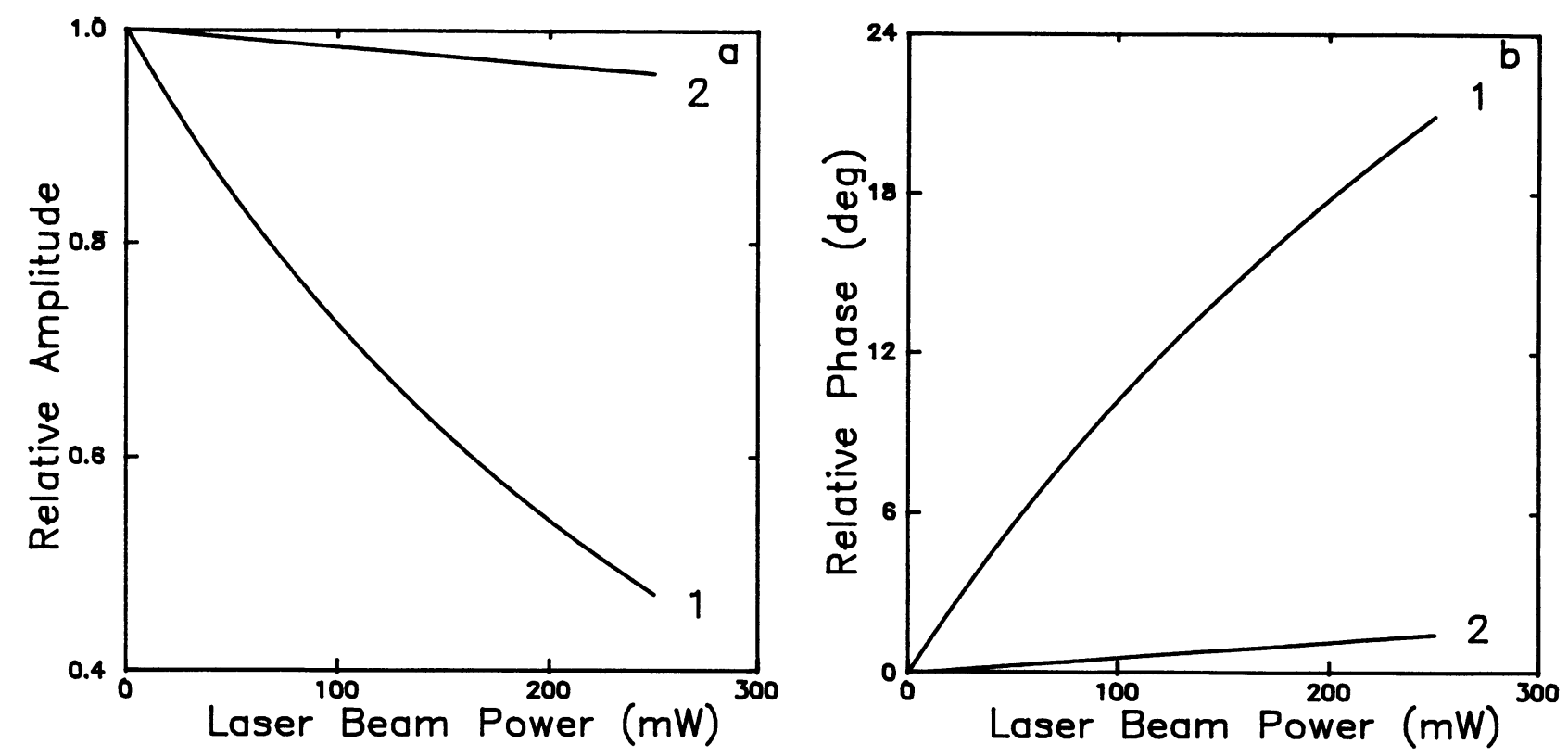

Fig. 6. - Relative OBD amplitude (a) and phase (b) calculated as a function of the heating beam power for $k_{\mathrm{s}}=1 \mathrm{~W} \cdot \mathrm{m}^{-1} \mathrm{~K}^{-1}$ (curve 1) and $k_{\mathrm{s}}=20 \mathrm{~W} \cdot \mathrm{m}^{-1} \mathrm{~K}^{-1}$ (curve 2). The values of the parameters are as follows : $a=200 \mu \mathrm{m}, z=200 \mu \mathrm{m}, B=5 \times 10^{5}$ and $T_{0}=300 \mathrm{~K}$.

\section{Conclusion.}

We have shown that the temperature rise in the deflecting fluid, resulting from a dc laser heating of the sample, may cause changes in the optical beam deflection. The reason for this is the temperature induced variations of the refractive index and thermal diffusivity of the fluid which define the deflection magnitude. One should bear in mind that such effects may play role, for example, when monitoring with OBD technique in situ laser beam annealing of semiconductors or phase transitions at high temperatures.

The use of an additional unmodulated laser beam for heating the sample allows one to study the influence of the dc heating on the mirage effect, regardless of the pump beam one. In case the temperature increase of the sample and the fluid, due to the pump beam heating, cannot be neglected, effects similar to those, described in this work, should be expected in the conventional OBD experiments. These effects are considered to play an important role, in (i) comparing samples with great difference in thermal conductivity coefficients; (ii) thermal diffusivity determination, when doing successive measurements at different probe beam offsets.

\section{Acknowledgment.}

The authors would like to thank prof. J. Pelzl who provided us with a position sensitive detector.

\section{References}

[1] Boccara A. C., Fournier D. and Badoz J., Appl. Phys. Lett. 36 (1980) 136.

[2] Jackson W. B., AMER N. M., Boccara A. C. and FoUrNIER D., Appl. Opt. 20 (1981) 1333.

[3] Aamodt L. C. and Murphy J. C., J. Appl. Phys. 52 (1981) 4903.

[4] Opsal J., Rosencwaig A. and Willenborg D., Appl. Opt. 22 (1983) 3169.

[5] Royce B. S. H. and Benziger J. B., IEEE Trans. Ultrason. Ferroelectr. Freq. Control UFFC-33 (1986) 561.
[6] Touloukian Y.S., Thermophysical Properties of Matter (Ed. New York : Plenum) 1970.

[7] Murphy J. C. and Aamodt L. C., J. Appl. Phys. 51 (1980) 4580.

[8] Carslow H. S. and Jaeger J. C., Conduction of Heat in Solids (Oxford U.P., London, 1959) Chapter 8.

[9] Mandelis A., J. Appl. Phys. 54 (1986) 3404. 